\title{
Todos ellos no son los que pueden justificarnos*
}

\author{
EDUARDO AlEJANDRO BARRIO \\ Departamento de Filosofía \\ Universidad de Buenos Aires \\ ebarrio@filo.uba.ar
}

\begin{abstract}
Resumen: En este artículo critico el enfoque de Luis Robledo acerca de la relación entre consenso global y justificación. Argumento que, contra lo que dice Robledo, no es concebible una situación en donde se produzca consenso global y carencia de justificación. Mi postura es que para que esa situación sea imaginable se requiere que el que advierte la carencia de justificación sea uno de los integrantes efectivos o potenciales de la comunidad que comparte las normas epistémicas. Para que haya carencia de justificación, no debe haberse cancelado el juego de dar y pedir razones, lo cual imposibilita que haya consenso global.
\end{abstract}

Palabras clave: comunidad, consenso, justificación, práctica

Desde hace mucho tiempo, el último teorema de Fermat es uno de los desafíos principales a los que se ha visto enfrentada la comunidad de los matemáticos. ${ }^{1}$ Subyace al teorema la idea de que es imposible separar el resultado de elevar al cubo un número en la suma de dos números, cada uno de los cuales ha sido elevado al cubo. Lo mismo sucede con cualquier potencia mayor que tres, pero no sucede con el cuadrado. Así, 25 es el resultado de elevar al cuadrado el número 5. Podemos encontrar dos números, 3 y 4, cuyos cuadrados respectivos son 9 y 16, cuya suma es el resultado de elevar al cuadrado al número 5 . ¿Tenemos una prueba de este teorema matemático? En su famosa nota al margen, Fermat escribió que contaba con tal justificación, aunque no nos dejó rastro de dicho procedimiento.

Supongamos que dos matemáticos japoneses anuncian a la comunidad matemática que han encontrado tal prueba. Supongamos que, luego de algunos estudios, la totalidad de los matemáticos cree que tal prueba es correcta. Supongamos entonces que hay un consenso global entre los matemáticos actuales acerca de que el último teorema de Fermat ha sido probado.

* Deseo destacar el apoyo brindado por la Fundación Antorchas (Argentina).

${ }^{1}$ Los personajes que aparecen en el texto son ficticios y cualquier semejanza con la historia reciente de la filosofía de las matemáticas es pura coincidencia. 
Supongamos que los epistemólogos analizan esa situación en el interior de la comunidad de matemáticos. En particular, ellos prestan atención a la existencia de elementos objetivos capaces de respaldar aquellos procesos epistémicos que dan lugar a la creencia de que algunos enunciados resultan justificados. Supongamos que casi todos están de acuerdo en que esos elementos existen y que no van a discutir con los relativistas que piensan que cualquier cosa puede justificarse. Supongamos también que la mayor parte son contextualistas en el sentido de que las normas que se siguen al obtener tal justificación son propias de cada comunidad. Es decir, que tales normas pudieron haber sido otras (y es posible que la comunidad las cambie en el futuro), aunque la comunidad confía en ellas porque son las mejores que han podido conseguir.

Supongamos también que un sector de los epistemólogos sostiene que el consenso global entre los matemáticos actuales es la mejor muestra de la existencia de esos elementos objetivos que respaldan la creencia de que el último teorema de Fermat finalmente ha sido probado. Tal idea se sustenta en que la totalidad de los matemáticos actuales han revisado una y otra vez el procedimiento efectuado por los dos matemáticos japoneses; han discutido y escuchado las razones de sus pares y finalmente se han convencido de que no hay ninguna razón para dudar. La totalidad de ellos se comportan como si aceptaran el último teorema de Fermat, y ese comportamiento nos autoriza a creer que ese teorema ha sido justificado en esa comunidad conforme a sus propias normas epistémicas. Según esta descripción, para justificar el último teorema de Fermat hay que producir un procedimiento que convenza a los restantes miembros de la comunidad. En una situación en que se produce consenso global, no existen más peticiones de razones; se clausura el juego del convencimiento y se genera una situación de consenso estable.

\section{III}

Supongamos que reviso la prueba de los matemáticos japoneses y encuentro un error. Supongamos que ese error es producto de una descuidada aplicación de un principio inferencial que la comunidad de matemáticos reconoce como propio. Supongamos que yo afirmara que, aunque exista un consenso entre todos los matemáticos, ellos no han justificado el último teorema de Fermat; han cometido un error colectivo al considerar que habían logrado probar el teorema. Según sus propias normas, no hay tal justificación a pesar del consenso logrado. De este hecho, Robledo ${ }^{2}$ (en la

${ }^{2}$ Cfr. L. Robledo, “iPuede realmente una mayoría estar equivocada?, pp. 143-149, en este número. 
línea de Putnam $)^{3}$ infiere que el concepto de justificación supone que lo justificado para una comunidad es lógicamente independiente del consenso comunitario porque, cuando nos preguntamos si el último teorema de Fermat está justificado, no estamos dispuestos a aceptar como una razón el hecho sociológico de que los miembros de la comunidad de matemáticos están dispuestos a aceptarlo. Aunque lo que haya sea sólo la conducta lingüística observable de la comunidad de matemáticos y aunque no podamos atribuirles coherentemente una norma epistémica que no emergiera de ella, según Robledo, parece concebible que yo considere la posibilidad de que todos ellos se encuentren radicalmente equivocados.

La estrategia argumentativa de Robledo parte, entonces, de la afirmación de que si se puede concebir una situación en la cual todos los integrantes de una comunidad dirían que una afirmación está justificada, pero en realidad de acuerdo con sus propias normas epistémicas no lo está, entonces lo que resulta justificado para una comunidad tiene que ser lógicamente independiente del consenso global comunitario. Como tal situación es concebible, Robledo concluye que hay algo que está más allá, hay algo que trasciende lo que dicen y hacen los miembros de una comunidad.

El punto, entonces, es debatir si tal situación es concebible. Lo primero que tenemos que advertir es la cuestión de cuál es el punto de vista desde donde es posible realizar esa narración. Según Cresto, ${ }^{4}$ esas condiciones requieren un observador externo. Robledo parece coincidir en este punto, aunque yo no estoy muy seguro de que esto sea así. La clave está en si en esa situación en la que advierto que la prueba del teorema de Fermat está mal, comparto o no con los matemáticos la misma práctica justificatoria. Robledo no niega que esto pueda ser así, con lo cual ellos y yo compartimos la misma concepción acerca de cuáles pasos son correctos y cuáles pasos son incorrectos en una demostración. A partir de este punto, es fácil concluir que, en un sentido obvio, soy parte de esa comunidad y que, por lo tanto, para no violar la coherencia narrativa, la historia requiere que, a diferencia de lo que Robledo supone, haya que eliminar la idea de que lo que había era un consenso global. No estaba lógicamente clausurado el juego de dar y pedir razones y lo que teníamos era un consenso inestable. Seguramente, si les muestro mi hallazgo, ellos se convencerán conmigo de que la prueba tenía un error, sin que por ello se necesite recurrir a normas inferenciales que estén más allá de nuestras propias prácticas inferenciales efectivas.

Lo que quiero plantear, entonces, es que se presenta una disyuntiva frente a esta situación. O bien formo parte de la comunidad, con lo cual descu-

${ }^{3}$ Cfr. H. Putnam, Realism with a Human Face, Harvard University Press, Cambridge, 1990, pp. 20 y ss.

${ }^{4}$ E. Cresto, "Justificar, interpretar y otros asuntos pragmáticos", pp. 151-158, en este número. 
bro un error, y lo cual muestra que hay que abandonar la prueba de los dos japoneses. En esta alternativa, todos (los matemáticos y yo) compartimos la misma práctica de justificar teoremas (aceptamos las mismas reglas para efectuar la prueba). De esto se sigue que formo parte de la comunidad y que no había consenso global; entonces, no se había clausurado el juego de dar y pedir razones. O bien, tal como sostiene Cresto, para asegurar la existencia de un consenso global, no debo formar parte de la comunidad. Esto tiene que presuponerse, si no estamos ante un caso obvio de abandono de una prueba que creíamos correcta pero que en realidad no lo era de acuerdo con los propios criterios epistémicos contextuales de una comunidad.

\section{IV}

Supongamos, entonces, que yo no formo parte de la comunidad de matemáticos. No soy uno de los miembros de la comunidad necesarios para asegurar el consenso. Robledo argumenta, ${ }^{5}$ frente a esta interpretación de la narración, que no podemos identificar práctica inferencial colectiva efectiva y práctica inferencial correcta. Su argumento es que, conforme a tal identificación, sería inconcebible un error colectivo como el de la comunidad de matemáticos, y sólo observando la comunidad desde afuera (tal como parece que lo hago yo), podríamos concebir esa situación. Tal cosa, argumenta Robledo, "viola el principio contextualista, según el cual cada comunidad tiene sus propias normas epistémicas, pues ahora sus normas no son propiamente las de los matemáticos, sino las que yo les atribuyo, que por hipótesis no son las mismas que ellos reconocen". ${ }^{6}$ Nuevamente aquí, la historia requiere que analicemos algunos aspectos para que continúe siendo narrativamente coherente (y para que, por lo tanto, sea una historia concebible). Lo que tiene que pasar para que yo no sea un integrante de la comunidad es que haya algunas maneras de justificar afirmaciones que los matemáticos y yo no compartimos. Supongamos que esto es así y que, de todas formas, advierto que, de acuerdo con las propias normas que ellos (y no yo) aplican al intentar justificar el último teorema de Fermat, el teorema no está justificado. Para que eso sea posible, tengo que realizar la contraprueba, de acuerdo con sus propias normas. Debo comportarme como si yo aceptara sus normas, y, al aplicarlas, descubrir el error. Pero si esas normas no son las mías, ¿de dónde saco esas normas si no es a partir de lo que ellos mismos dicen? Supongamos que aplico las normas epistémicas que ellos dicen que aplican. Supongamos que de esa aplicación surge un error en la prueba de los japoneses. Tal cosa muestra

${ }^{5}$ L. Robledo, op. cit.

${ }^{6}$ Cfr. L. Robledo op. cit., p. 147. 
que hay un integrante posible de la comunidad de matemáticos, que tiene aún razones que presentar contra la prueba de los japoneses. En ese caso, nuevamente, no tenemos consenso global y, por lo tanto, se trata de un caso de error de aplicación de las normas que regulan la práctica de justificar. Robledo tendría que mostrar que aun cuando yo les muestre el error de acuerdo con sus propias normas, ellos podrían tener razones para seguir aceptando la prueba de los japoneses. Nótese que siempre es fácticamente posible que ellos sigan aceptando la prueba. Como sostiene Díaz Legaspe, ${ }^{7}$ ellos podrían ser una comunidad de caprichosos matemáticos que se niegan a aceptar las razones que alguien, de acuerdo con las propias normas racionales de esa comunidad, pudiera presentarles para mostrar que su comportamiento no es el correcto. ¿Cómo describiríamos esta situación? Seguramente diríamos que esa comunidad, de acuerdo con sus propias normas, se está comportando irracionalmente. No diríamos, tal como lo necesita Robledo, que tendríamos que abandonar la idea de que las normas están en la conducta efectiva de la comunidad. Lo que diríamos es que la conducta es irracional aun con sus propios principios normativos.

Supongamos que la comunidad de matemáticos tiene en su propio lenguaje recursos expresivos suficientes como para expresar sus propias normas en un lenguaje accesible para mí.

Según Robledo, esta suposición, junto con la idea de identificar lo correcto con la práctica comunitaria efectiva, nos conduce a una situación paradójica. El error que yo les señalo de acuerdo con sus propias normas muestra que la justificación es independiente de lo que dicen. Si esto es así, argumenta Robledo, "con el fin de negar que sea concebible que [los matemáticos] pueda[n] estar globalmente [equivocados] respecto de la justificación [del último teorema de Fermat], es necesario afirmar que [ellos] puede[n] estar globalmente equivocad[os] respecto de cuáles son sus normas [epistémicas]". ${ }^{8} \mathrm{Mi}$ disentimiento con Robledo en este punto es que ellos no se equivocan respecto de cuáles son las normas epistémicas. Como dije antes, si no tengo en cuenta cuáles son las normas que ellos dicen que aplican y si esas normas, para que yo no sea parte del consenso global, no son las mías, ¿de dónde puedo sacar yo que ésas y no las que dicen que aplican son las normas de la comunidad de matemáticos? Sostener que las normas no trascienden las prácticas inferenciales efectivas no quiere decir que un grupo, incluso un grupo numeroso, no pueda equivocarse al aplicarlas. Por supuesto, esa situación es improbable; pero no imposible. El caso

${ }^{7}$ J. Díaz Legaspe, “Consenso, justificación y pragmatismo”, pp. 159-165, en este mismo número.

${ }^{8}$ Cfr. L. Robledo, op. cit., p. 147-148. 
es, sin embargo, que ésta no es la situación que Robledo necesita concebir. Desde mi punto de vista, aun aplicando sus normas, ellos se equivocan al seguirlas. ¿De dónde saco yo que se equivocan? De lo que ellos dicen. Para que haya error tiene que haber alguna norma o criterio desde los cuales la práctica epistémica resulte equivocada. No tiene sentido la idea de que ellos crean estar siguiendo una norma o la idea de que ellos digan que están siguiendo una norma, cuando ninguno de ellos la está siguiendo. Pero hay alguien - yo- que aplicando las normas advierte el error. Si ellos son racionales, dejarán de aceptar la prueba. Y esto no significa que las normas sean trascendentes a la práctica efectiva. Simplemente significa que no estamos hablando de la mera situación en la que todos se comportan como si aceptaran una afirmación. Para lograr un consenso global, nadie debe poder dar una razón adicional contra la prueba. Sólo en este caso, la justificación no es independiente del consenso global.

\section{VI}

Supongamos que estoy de acuerdo con la idea de que las normas epistémicas no trascienden las prácticas efectivas. Que ellas son inseparables de tales prácticas en el sentido de que no es posible confrontarlas con ningún curso de acción, de manera tal que la corrección de cada una de las acciones involucradas en tal curso no es el resultado de haberlas interpretado a la luz de esas normas. La corrección o incorrección de la prueba efectuada por los dos japoneses no puede reducirse a la confrontación entre las normas inferenciales supuestamente involucradas y sus pasos probatorios. Supongamos que sólo a la luz de la práctica probatoria aceptada por la comunidad matemática tiene sentido hablar de corrección de una prueba. Ellos, los que participan de esa práctica, dominan lo que resulta apropiado inferir. Por supuesto, este dominio inferencial no debe pensarse subjetivamente. No es simplemente lo que a cada uno le parece que tiene que inferir; tampoco es lo que regularmente hace la comunidad. No es la mera regularidad entre lo que infieren y lo que dicen que infieren lo que asegura la corrección. La corrección debe buscarse en las condiciones normativas que constituyen la práctica como tal.

Supongamos que considero que cada vez que se realiza una prueba correctamente surge consenso en la comunidad. Que los integrantes de esa comunidad, si se comportan racionalmente, están dispuestos a aceptar la prueba. En este sentido, estoy sosteniendo que el consenso se produce a partir de la existencia de una prueba correcta. Por supuesto, para cualquiera que advierta que existe tal actitud colectiva frente a una afirmación, esto será un elemento para reconocer la existencia de una prueba correcta; pero no es lo que constituye la corrección de la prueba. Puede haber consensos producidos por otros medios, consensos que no surjan de la correcta 
aplicación de las normas constitutivas de la comunidad. Lo que argumento, contra Robledo, es que nunca podremos concebir un consenso global racional.

\section{VII}

En suma, la situación de Robledo no parece concebible siguiendo con las restricciones que él mismo aceptaría. Si la justificación es siempre relativa a una comunidad, no es posible una situación en la cual haya consenso global y se carezca de justificación. Buscar la corrección en las prácticas comunitarias no supone la imposibilidad del error; tan sólo limita las posibilidades del error masivo. Tampoco es plausible suponer que las normas sean otras que las que los participantes de una práctica dicen que son. Por supuesto, esto tampoco significa que lo que constituye la corrección, el patrón normativo, esté dado por la expresión de esa norma. No hay ningún punto en el que confrontar la práctica con la formulación de la norma.

Recibido el 19 de junio de 2002; aceptado el 26 de noviembre de 2002 